\title{
The use of light-emitting diodes to stimulate mitochondrial function and liver regeneration of partially hepatectomized rats
}

T. Castro-e-Silva ${ }^{1}$,

O. Castro-e-Silva ${ }^{3}$, C. Kurachi ${ }^{1}$, J. Ferreira ${ }^{2}$,

S. Zucoloto ${ }^{2}$ and V.S. Bagnato ${ }^{1}$

\author{
1'Instituto de Física de São Carlos, Universidade de São Paulo, \\ São Carlos, SP, Brasil \\ ${ }^{2}$ Departamento de Patologia and ${ }^{3}$ Departamento de Cirurgia e Anatomia, \\ Faculdade de Medicina de Ribeirão Preto, Universidade de São Paulo, \\ Ribeirão Preto, SP, Brasil
}

Correspondence

T. Castro-e-Silva

Rua Campos Salles, 890/92

15015-110 Ribeirão Preto, SP

Brasil

E-mail: castroesilva@pg.ffclrp.usp.br

Research supported by FAPESP and CNPq.

$\ldots \ldots \ldots \ldots \ldots \ldots \ldots$

Received October 17, 2006 Accepted May 18, 2007

\begin{abstract}
The biostimulating effect of laser radiation has been observed in many areas of Medicine. However, there are still several questions to be answered, among them the importance of light coherence in the stimulatory process. In the present study, we used light-emitting diodes (LED) to promote the stimulation of liver regeneration after partial hepatectomy in rats. Fourteen male Wistar rats weighing 200$250 \mathrm{~g}$ were submitted to partial hepatectomy $(70 \%)$ followed by LED light irradiation $(630 \mathrm{~nm})$ of the remaining part of the liver at two doses, i.e., $10(\mathrm{~N}=7)$ and $140(\mathrm{~N}=7) \mathrm{J} / \mathrm{cm}^{2}$. A group irradiated with laser, $590 \mathrm{~nm}\left(\mathrm{~N}=7,15 \mathrm{~J} / \mathrm{cm}^{2}\right)$ was performed for the study of proliferating cell nuclear antigen-labeling index. Data are reported as mean \pm SEM. Statistical comparisons of the groups were performed by analysis of variance for parametric measurements followed by the Bonferroni post-test, with the level of significance set at $\mathrm{P}<0.05$. Respiratory mitochondrial activity was increased in the irradiated groups (states 3 and $4 ; \mathrm{P}<0.05$ ), with better results for the group exposed to the lower LED dose $\left(10 \mathrm{~J} / \mathrm{cm}^{2}\right)$. The proliferating cell nuclear antigen-labeling index, by immunohistochemical staining, was similar for both LED-exposed groups $(\mathrm{P}>0.05)$ and higher than for the control group $(\mathrm{P}<0.05)$. The cell proliferation index obtained with LED and laser were similar $(\mathrm{P}>0.05)$. In conclusion, the present results suggest that LED irradiation promotes biological stimulatory effects during the early stage of liver regeneration and that LED is as effective as laser light, independent of the coherence, divergence and cromaticity.
\end{abstract}

Key words

- Liver

- Mitochondria

- Laser

- Light-emitting diode

- Liver regeneration

- Proliferating cell nuclear antigen
Many studies have demonstrated that laser light can interfere in distinct ways with cellular metabolism (1). In particular, liver regeneration has been a recent focus of investigation (2). Both visible light and infra- red light have been shown to have many different effects inside the cell (3). Light radiation must be absorbed to promote a biological response (4) and, on this basis, we expected any type of light therapy to be as 
effective as light itself, and not a function of coherence which is a unique property of laser light. Thus, almost monochromatic light is expected to present effects similar to those of laser. With this in mind, we performed a series of experiments to demonstrate that the effects of low-energy laser therapy are due to the effects of light and not to the unique properties of lasers. Recently, a light-emitting diode (LED)-based device has been used for the treatment of skin tumors by photodynamic therapy instead of laser radiation, and the success obtained has shown the potential of such device for different types of light therapy as well (5). LED has a considerably narrow emission spectrum, but the main difference between LED and lasers is that fact that LED is not a coherent radiation (6). The absence of coherence in the light used for biostimulation is still a subject under debate. In the present study, we investigated for the first time cell proliferation and mitochondrial respiratory function after irradiation with LED. Our main purpose was to determine if the LED device can produce effects similar to those obtained with laser in previous studies by our group $(2,3)$. Our results indicate the efficacy and similarities of LED and laser light, with the advantage that LEDs are economically more convenient and much less restrictive to operate. Besides, LEDs permit the construction of devices anatomically adapted to the point of application.

LEDs and lasers both produce radiation at specific wavelengths. Nevertheless, LEDs are neither coherent nor collimated and are broader in emission when compared with lasers, with these properties permitting higher penetration in many cases. We have used a home-prepared special LED, Brazilian patent, 2002 (7), that employs an array of emitting centers, with wavelength centered at $630 \mathrm{~nm}$. The overall emitted power over a full hemisphere is about $500 \mathrm{~mW}$, given an energy density that depends on the distance between the device and the target. Intensities of 20 to $50 \mathrm{~mW} / \mathrm{cm}^{2}$ are obtained with this device.

Male Wistar rats weighing 200-250 g were obtained from the Central Animal House of the Faculty of Medicine of Ribeirão Preto, University of São Paulo, Brazil. The animals received standard laboratory chow (Purina) and water ad libitum, and were kept in identical housing units on a 12-h light/ dark cycle. Two groups of 7 rats each were exposed to LED, and one unexposed group was used for control $(N=7)$. Seventy percent partial hepatectomy was performed by the method of Higgins and Anderson (8). Briefly, under diethylether anesthesia, the peritoneal cavity was entered through a median incision, and the left lateral and median lobes of the liver were excised. After the operation, blood clots were thoroughly wiped with gauze, and the wound was closed by layers with running 3-0 silk sutures.

Before closure of the surgical wound, the two groups of animals prepared for LED irradiation were treated by direct illumination of the remaining liver with a dose of 10 $\mathrm{J} / \mathrm{cm}^{2}$ (LED 10) for $3 \mathrm{~min}$ and $140 \mathrm{~J} / \mathrm{cm}^{2}$ (LED 140) for $40 \mathrm{~min}$. The laser group was treated by direct irradiation of the remaining liver with a dose of $15 \mathrm{~J} / \mathrm{cm}^{2}$ for $5 \mathrm{~min}$. The laser employed was a dye laser pumped by an argon ion laser, producing a light beam at $590 \mathrm{~nm}$. The control group was submitted to the same partial hepatectomy but was not exposed to LED or laser.

Twenty-four hours after the procedure, the LED, laser and control groups were sacrificed under diethylether anesthesia $(2,3)$. Samples of liver tissue and blood from the inferior vena cava were collected for the determinations described below.

Immunohistochemical staining for proliferating cell nuclear antigen (PCNA) of the formalin-fixed and paraffin-embedded liver tissues was performed with a monoclonal primary anti-PCNA antibody (PCNA, PC10; Dako A/S, Glostrup, Denmark) as previously described (9). Sections of $4 \mu \mathrm{m}$ were cut, mounted on poly-L-lysine-coated glass 
slides, deparaffinized, rehydrated in an increasing alcohol series, placed in PBS, and treated with $2 \%$ hydrogen peroxide in methanol for 15 min to block endogenous peroxidase activity. Nonspecific protein binding was blocked by preincubation with 5\% normal horse serum diluted in PBS for $30 \mathrm{~min}$. This was followed by incubation with the PC-10 monoclonal primary antibody diluted 1:40 in PBS for $120 \mathrm{~min}$ at $25^{\circ} \mathrm{C}$. The sections were then incubated for $35 \mathrm{~min}$ with a biotinylated horse anti-mouse immunoglobulin. The reaction product was detected with an avidin-biotin-peroxidase complex and diaminobenzidine was used as a chromogen substrate. Positive and negative controls were used to assess and control for the staining procedure. Sections were examined blindly at high power (400X), and 10 fields were chosen at random. Nuclear-labeling indices for PCNA (positive nuclei/total number of counted nuclei) were determined by evaluation of at least 1000 hepatocyte nuclei.

Isolation of liver mitochondria was performed by differential centrifugation as previously described (10). Mitochondrial oxygen consumption was determined polarographically with a home-made oxygraph equipped with a Clarck oxygen electrode. The respiratory parameters were determined according to an established procedure (11). Succinate $(5 \mathrm{mM})$ was used as an oxidizable substrate in $1.4 \mathrm{~mL}$ of medium with $125 \mathrm{mM}$ sucrose, $65 \mathrm{mM} \mathrm{KCl}, 1 \mathrm{mM} \mathrm{MgCl} 2,2 \mathrm{mM}$ $\mathrm{KH}_{2} \mathrm{PO}_{4}, 0.1 \mathrm{mM}$ EGTA, and $10 \mathrm{mM}$ HEPES-KOH, pH 7.4, and $2.0 \mathrm{mg}$ mitochondrial protein was used. State 3 respiration was induced with $400 \mathrm{nmol} \mathrm{MgADP}$, and state 4 respiration (basal mitochondrial respiration) was determined after phosphorylation of additional ADP (10). The ratio between state 3 and state 4 rates (respiratory control ratio), which represents coupling between electron transport and oxidative phosphorylation, was determined. Mitochondrial protein content was determined by the biuret method (12).

The electrical transmembrane potential difference $(\Delta \psi)$ was monitored spectrofluorimetrically using $5 \mu \mathrm{M}$ safranine $\mathrm{O}$ as an indicator and an SLM-Aminco Bowman, series 2 luminescence spectrophotometer (Urbana, IL, USA) at the 495/586 nm excitation/emission wavelength pair. Assays were performed in an incubation medium containing $200 \mathrm{mM}$ sucrose, $1 \mathrm{mM} \mathrm{MgCl} 2,2.5$ $\mu \mathrm{M} \mathrm{NaH} \mathrm{PO}_{4}$ and $10 \mathrm{mM}$ HEPES-NaOH, $\mathrm{pH}$ 7.4. Mitochondria were energized with 5 $\mathrm{mM}$ sodium succinate. The membrane potential variation is reported as $\mathrm{mV}$ as usual (11). Data are reported as mean \pm SEM. Statistical comparisons of the groups were performed by analysis of variance (ANOVA) for parametric measurements followed by the Bonferroni post-test, with the level of significance set at $\mathrm{P}<0.05$.

Figure 1 illustrates the results of state 3 (1A) and state 4 respiration (1B) and the respiratory control ratio (1C) for the three main groups of hepatectomized and treated animals. The group treated with a LED dose of $10 \mathrm{~J} / \mathrm{cm}^{2}$ showed better state 3 and 4 values when compared with the other groups. Since state 3 (1A) represents the efficiency of mitochondrial respiration, there was a clear enhancement caused by exposure to LED. Again, the increase of about $20 \%$ in the absolute value of state 3 agrees with our previous observations when laser light was used and state 3 was observed $24 \mathrm{~h}$ after partial hepatectomy $(2,3)$. This biostimulating effect caused by LED is also demonstrable in other mitochondrial respiratory functions. Figure 1B shows state 4 of the mitochondrial respiratory process. The increase of state 4 indicates an elevation of ionic flow through the inner mitochondrial membrane caused by light exposure, a result that agrees with the previously observed enhancement of state 4 due to laser exposure at a similar energy level $(2,3)$. The membrane potential was measured in all three groups and the results are presented in Figure 1B. The same 
values for all groups indicate that the procedure with LED illumination does not cause any extra effect on the membranes, whose integrity was preserved during illumination.

Liver regeneration was estimated by counting the stained nuclei in the PCNA immunohistochemical assay (Figure 1E). The 1.5-fold increase in the labeling index for the LED groups was remarkable, showing the irradiation effect on hepatocyte proliferation compared to control. Both irradiated groups showed greater liver regeneration compared to control. Comparing the two groups exposed to different light doses, we
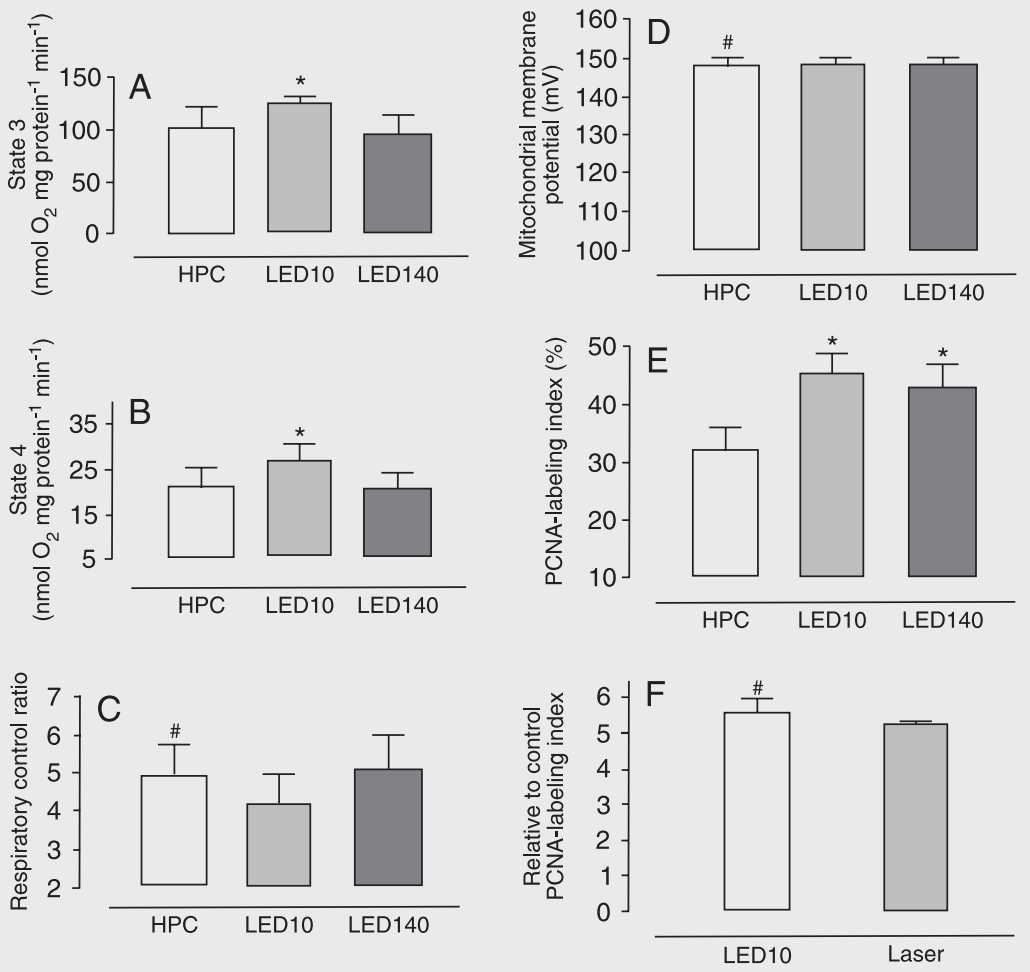

Figure 1. Effect of light-emitting diode (LED) and laser light irradiation on rats with partial hepatectomy. All panels contain data for control, LED $10 \mathrm{~J} / \mathrm{cm}^{2}$ (LED10) and LED $140 \mathrm{~J} / \mathrm{cm}^{2}$ (LED140), except panel $F$ which compares LED10 and laser. Each group consisted of 7 rats in each group. $A$, Mitochondrial state $3 .{ }^{*} \mathrm{P}<0.05$ for LED10 vs LED140 or hepatectomy control (HPC). B, Mitochondrial state 4. ${ }^{*} \mathrm{P}<0.05$ for LED10 vs LED140 or HPC. C, Respiratory control ratio. \#P $>0.05$ for HPC vs LED10 and LED140. D, Mitochondrial membrane potential. \#P $>0.05$ for HPC vs LED10 and LED140. E, Proliferating cell nuclear antigen (PCNA)-labeling index. Comparison of HPC vs LED10 and LED140. ${ }^{*} \mathrm{P}<0.05$ for LED10 and LED140 vs HPC. Statistical comparisons were carried out with ANOVA followed by the Bonferroni post-test. $F$, Values of the PCNA-labeling index (relative to control PCNA-labeling index - data normalized to the dose) for the LED10 and laser $\left(15 \mathrm{~J} / \mathrm{cm}^{2}\right)$ groups. $\#$ P $>0.05$ for LED10 vs laser. observed that LED10 presented greater mitochondrial function in state 3 , which was also followed by an increase in state 4 respiration. In the LED140 group the increase of state 3 and state 4 respiration was not so dramatic and the values were close to control. This type of behavior is common in biostimulation with light, in which inhibition effects have also been observed (13). Finally, we compared the effect of LED and laser light concerning cell proliferation. In a previous study by our group (3) we employed laser irradiation to measure the labeling index after the same type of hepatectomy as used in the present study. The laser used in the previous study (3) had a wavelength of $630 \mathrm{~nm}$, the same as the peak of LED emission used here. In that case, a similar investigation was performed. To show a possible equivalence of the two light sources, we present in Figure $1 \mathrm{~F}$ a comparison of the PCNA obtained with them. Since a slight difference in dose was used for laser and for LED, the comparison is more adequate if we present the data normalized to the dose. This type of procedure can be justified based on the fact that the dose represents the overall energy delivered and, therefore, the total amount of biomolecule excitation one can effectively achieve.

The data in Figure $1 \mathrm{~F}$ were obtained by normalizing the real PCNA value to the respective control, with the result being divided by the dose used. Only the data obtained with $10 \mathrm{~J} / \mathrm{cm}^{2}$ for LED and $15 \mathrm{~J} / \mathrm{cm}^{2}$ for laser (3) were considered. With this comparison we observed that not only the qualitative behavior of biostimulation using LED or laser is similar, but also that the quantitative results were also closely similar. Laser and LED share the fact that they emit light although the characteristics of the light have very little in common. Coherence, monochromaticity and divergence are some of them. However, for biostimulation of metabolic reactions, these properties are not so essential. Because of the scattering proper- 
ties of tissues, coherence is destroyed, and because of the broad band absorption of chromophores, monochromaticity is within limits of absorption. Therefore, in terms of interaction with tissue biochemistry, both should work in a quite equivalent way and this is in fact what we documented here.

This is not such a surprising observation since what matters in the use of light stimulation is the capacity to interact with the biomolecules more than the coherence present in the laser light. The real influence of coherence properties in low-intensity laser therapy is a subject still under debate. Nevertheless, our results show that, concerning liver regeneration stimulated with light, coherence does not seem to play an important role.

The present results show that LED promotes biological stimulatory effects in the early stage of liver regeneration without any detectable damage to cell function. The influence of non-coherent light on mitochondrial activity may be an important issue regarding the therapeutical uses of laser light compared to other light sources. This is a question constantly raised in the literature, but the answer has not yet been fully formulated.

\section{References}

1. Karu T. Photobiology of low-power laser effects. Health Phys 1989; 56: 691-704.

2. de Castro e Silva Junior O, Zucoloto S, Menegazzo LA, Granato $R G$, Marcassa LG, Bagnato VS. Laser enhancement in hepatic regeneration for partially hepatectomized rats. Lasers Surg Med 2001; 29: 73-77.

3. Castro-e-Silva O Jr, Zucoloto S, Marcassa LG, Marcassa J, Kurachi $\mathrm{C}$, Melo CA, et al. Spectral response for laser enhancement in hepatic regeneration for hepatectomized rats. Lasers Surg Med 2003; 32: 50-53.

4. Melo CA, Lima AL, Brasil IR, Castro e Silva O Jr, Magalhaes DV, Marcassa LG, et al. Characterization of light penetration in rat tissues. J Clin Laser Med Surg 2001; 19: 175-179.

5. Kurachi C, Melo CS, Marcassa LG, Zílio S, Bagnato VS, Cestari Filho GA, et al. Implantação clínica da terapia fotodinâmica no Brasil: breve histórico e resultados alcançados. Rev Soc Bras Cancerol 2002; 20: 33-41.

6. Stahl F, Ashworth SH, Jandt KD, Mills RW. Light-emitting diode (LED) polymerisation of dental composites: flexural properties and polymerisation potential. Biomaterials 2000; 21: 1379-1385.
7. Patent No. PI0200200-0. Instituto Nacional de Propriedade Industrial (INPI), Brazil; 2002.

8. Higgins GM, Anderson RM. Experimental pathology of the liver: I. Restoration of the liver of the white rat following partial surgical removal. Arch Pathol 1931; 12: 186-202.

9. Ramalho FS, Ramalho LN, Castro-e-Silva Junior O, Zucoloto S, Correa FM. Effect of angiotensin-converting enzyme inhibitors on liver regeneration in rats. Hepatogastroenterology 2002; 49: 13471351.

10. Souza ME, Polizello AC, Uyemura SA, Castro-Silva O, Curti C. Effect of fluoxetine on rat liver mitochondria. Biochem Pharmacol 1994; 48: 535-541.

11. Jordani MC, Santos AC, Prado IM, Uyemura SA, Curti C. Flufenamic acid as an inducer of mitochondrial permeability transition. Mol Cell Biochem 2000; 210: 153-158.

12. Kaplan RS, Pedersen PL. Characterization of phosphate efflux pathways in rat liver mitochondria. Biochem J 1983; 212: 279-288.

13. Morimoto $\mathrm{Y}$, Arai T, Kikuchi M, Nakajima S, Nakamura H. Effect of low-intensity argon laser irradiation on mitochondrial respiration. Lasers Surg Med 1994; 15: 191-199. 